\title{
A relação médico-paciente frente à telemedicina
}

\author{
The doctor-patient relationship with telemedicine \\ La relación médico-paciente con la telemedicina
}

Sílvia Cristina Marreiros de Carvalho Leite ${ }^{1 *}$, Brunna Marcela Nunes Leal ${ }^{1}$, Luciana Souza de Sousa', Dara Maria Pontes Gomes ${ }^{1}$, Sâmela Victória dos Santos Dias ${ }^{1}$, Marília Gabriela Dias Nery ${ }^{1}$, Deborah Ozima Mota Aroso ${ }^{1}$, Matheus Luiz Santos de Aguiar ${ }^{1}$, Renata Paula Lima Beltrão ${ }^{1}$, Augusto César Beltrão da Silva ${ }^{1}$.

\section{RESUMO}

Objetivo: Analisar a influência da telemedicina na relação médico-paciente. Métodos: Tratou-se de uma revisão integrativa de literatura. As bases de dados utilizadas para a busca foram a PubMed e Scientific Electronic Library Online (SCIELO). Os critérios de inclusão foram: Artigos científicos disponíveis na integra, publicados nos últimos dez anos (2010 a 2020), e pesquisa realizada com seres humanos, pesquisas com temática de acordo com os objetivos desse estudo. Os critérios de exclusão foram outras formas de publicação que não fossem artigos científicos completos, publicações com mais de dez anos (2010 a 2020), e pesquisas realizadas com animais ou in vitro. Resultados: Foram selecionados 12 artigos provenientes das bases SCIELO e PubMed, em sua maioria os estudos enfatizaram a telemedicina e a relação médicopaciente, e também a influência da telemedicina na assistência, uma vez que em meio a pandemia de Covid19 os canais e mídias de saúde são cada vez mais utilizados Considerações finais: A telemedicina é uma ferramenta que contribui para a aproximação do médico com o paciente, especialmente em tempos de pandemia, como a da COVID-19, em que o contato físico social teve que ser restringido.

Palavras-Chave: Telemedicina, Relações médico-paciente, Tecnologia da informação.

\begin{abstract}
Objective: Analyze the influence of telemedicine on the doctor-patient relationship. Methods: It was an integrative literature review. The databases used for the search were PubMed and Scientific Electronic Library Online (SCIELO). The inclusion criteria were: Scientific articles available in full, published in the last ten years (2010 to 2020), and research carried out with human beings, research with thematic according to the objectives of this study. The exclusion criteria were forms of publication other than complete scientific articles, publications older than ten years (2010 to 2020), and research carried out with animals or in vitro. Results: 12 articles were selected from the SCIELO and PubMed databases, most of the studies emphasized telemedicine and the doctor-patient relationship, and also the influence of telemedicine in care, since in the midst of the Covid-19 pandemic the channels and health media are increasingly used. Final considerations: Telemedicine is a tool that contributes to the approach of the doctor to the patient, especially in times of pandemic, such as that of COVID-19, in which physical social contact had to be restricted.
\end{abstract}

Keywords: Telemedicine, Doctor-patient relations, Information technology.

\section{RESUMEN}

Objetivo: Analizar la influencia de la telemedicina en la relación médico-paciente. Métodos: Fue una revisión integradora de la literatura. Las bases de datos utilizadas para la búsqueda fueron PubMed y Scientific Electronic Library Online (SCIELO). Los criterios de inclusión fueron: Artículos científicos disponibles

1 Instituto de Educação Superior do Vale do Parnaíba (IESVAP), Parnaíba - PI.

*E-mail: silviacmleite95@gmail.com

SUBMETIDO EM: 10/2020 
íntegramente, publicados en los últimos diez años (2010 a 2020), e investigaciones realizadas con seres humanos, investigación con temática acorde a los objetivos de este estudio. Los criterios de exclusión fueron formas de publicación distintas de los artículos científicos completos, publicaciones con más de diez años (2010 a 2020) e investigaciones realizadas con animales o in vitro. Resultados: Se seleccionaron 12 artículos de las bases de datos SCIELO y PubMed, la mayoría de los estudios enfatizó la telemedicina y la relación médico-paciente, y también la influencia de la telemedicina en la atención, ya que en medio de la pandemia Covid-19 los canales y los medios de salud son cada vez más utilizados Consideraciones finales: La telemedicina es una herramienta que contribuye al acercamiento del médico al paciente, especialmente en épocas de pandemia, como la del COVID-19, en la que había que restringir el contacto físico social.

Palabras clave: Telemedicina, Relaciones médico-paciente, Tecnología de la información.

\section{INTRODUÇÃO}

A medicina é a ciência que trata o ser humano em seus aspectos físico, social e emocional. Devido a abrangência da personalidade humana, cada paciente possui suas próprias necessidades que vão muito além da cura física, necessitando de atendimento individualizado e que supra suas expectativas, para tal a comunicação é imprescindível nesse processo de atendimento (BATISTA NA e LESSA SS, 2019).

A relação médico-paciente é pautada na comunicação, o profissional deve ser cordial e ter empatia pela situação que o paciente está enfrentando para conseguir estabelecer suas primeiras impressões sobre as necessidades emocionais e sociais envoltas no processo de adoecimento que acomete o bem-estar do indivíduo. Além disso, é a partir das primeiras conversas que o paciente vai se sentir confortável ou não para contar sobre seus anseios e problemas de saúde (ALMEIDA LP, et al., 2019).

A relação médico-paciente deve transmitir credibilidade e segurança ao paciente, e ser construída a partir da escuta qualificada e do acolhimento, pois essa relação é a base para o sucesso de toda assistência a saúde, uma vez que o paciente que se sente confiante e familiarizado com o profissional que o atende tende a colaborar mais e seguir corretamente as recomendações médicas (SANTANA S e PEREIRA AS, 2007).

Enxergar o paciente como pessoa e não como doença é o primeiro passo para estabelecer uma relação médico-paciente humanizada. As tecnologias de saúde surgem nesse contexto como ferramenta para aproximação do médico com o paciente, e em tempos que o distanciamento social passou a ser crucial devido a pandemia da Covid-19, a telemedicina vem sendo cada vez mais utilizada (KOGA RCR, et al., 2010).

A telemedicina consiste no uso das tecnologias da informação e comunicação para prestar atendimento a distância, garantindo segurança e a saúde do profissional e do paciente, e levando informações de maneira rápida e eficaz, além de fornecer atendimento médico a regiões com falta de profissionais (ARANTES ACC, et al., 2013).

Assim com base no exposto foi formulada a questão norteadora: Quais evidencias cientificas presentes na literatura sobre a relação médico-paciente frente a telemedicina? Para tal, esse estudo teve como objetivo: Analisar a influência da telemedicina na relação médico-paciente.

\section{MÉTODOS}

Esse estudo se tratou de uma revisão integrativa de literatura, elaborada com base na literatura vigente referente à relação médico-paciente na telemedicina, com o intuito de apresentar uma abordagem descritiva sobre o tema abordado. Para elaboração da pesquisa foi utilizado a combinação de descritores obtidos nos Descritores em Ciências da Saúde (DeCS) com o operador booleano de adição "AND", sendo esses descritores: Telemedicina; Relações Médico-Paciente; Tecnologia da Informação.

As bases de dados utilizadas para a busca foram a PubMed e Scientific Electronic Library Online (SCIELO), por serem bases com uma ampla variedade de revistas nacionais e internacionais, e permitirem o acesso gratuito aos estudos na integra.

REAS/EJCH | Vol.13(2) | e5694 | DOI: https://doi.org/10.25248/reas.e5694.2021 Página 2 de 9 
A pesquisa ocorreu entre os meses de setembro de 2020 a outubro de 2020. Para selecionar os artigos de cada base de dados foram utilizados critérios de inclusão e exclusão dos estudos, de acordo com os objetivos dessa revisão bibliográfica.

Assim, foram estabelecidos como critério de inclusão: Artigos científicos disponíveis na integra com acesso gratuito de seu conteúdo, publicados nos últimos dez anos (2010 a 2020), e pesquisa realizada com seres humanos, pesquisas com temática de acordo com os objetivos desse estudo.

Foram estabelecidos com critérios de exclusão outras formas de publicação que não fossem artigos científicos, artigos científicos incompletos ou que não tivessem acesso gratuito ao conteúdo, publicações com mais de dez anos (2010 a 2020), e pesquisas realizadas com animais ou in vitro, e artigos que fugissem da temática "telemedicina na frente a relação médico-paciente".

Assim, com base na estratégia de busca geral foram encontrados 14 artigos na base SCIELO, 11 artigos na base PubMed, totalizando 25 artigos. Todavia, aplicando os critérios de inclusão e exclusão, e realizando a leitura seletiva dos artigos e excluindo os artigos repetidos nas bases de dados, apenas 12 artigos foram condizentes com os objetivos desse estudo, sendo 8 provenientes da base SCIELO, 4 da base PubMed, conforme exposto no fluxograma abaixo (Figura 1).

Figura 1 - Fluxograma do processo de seleção dos estudos, $n=12$.

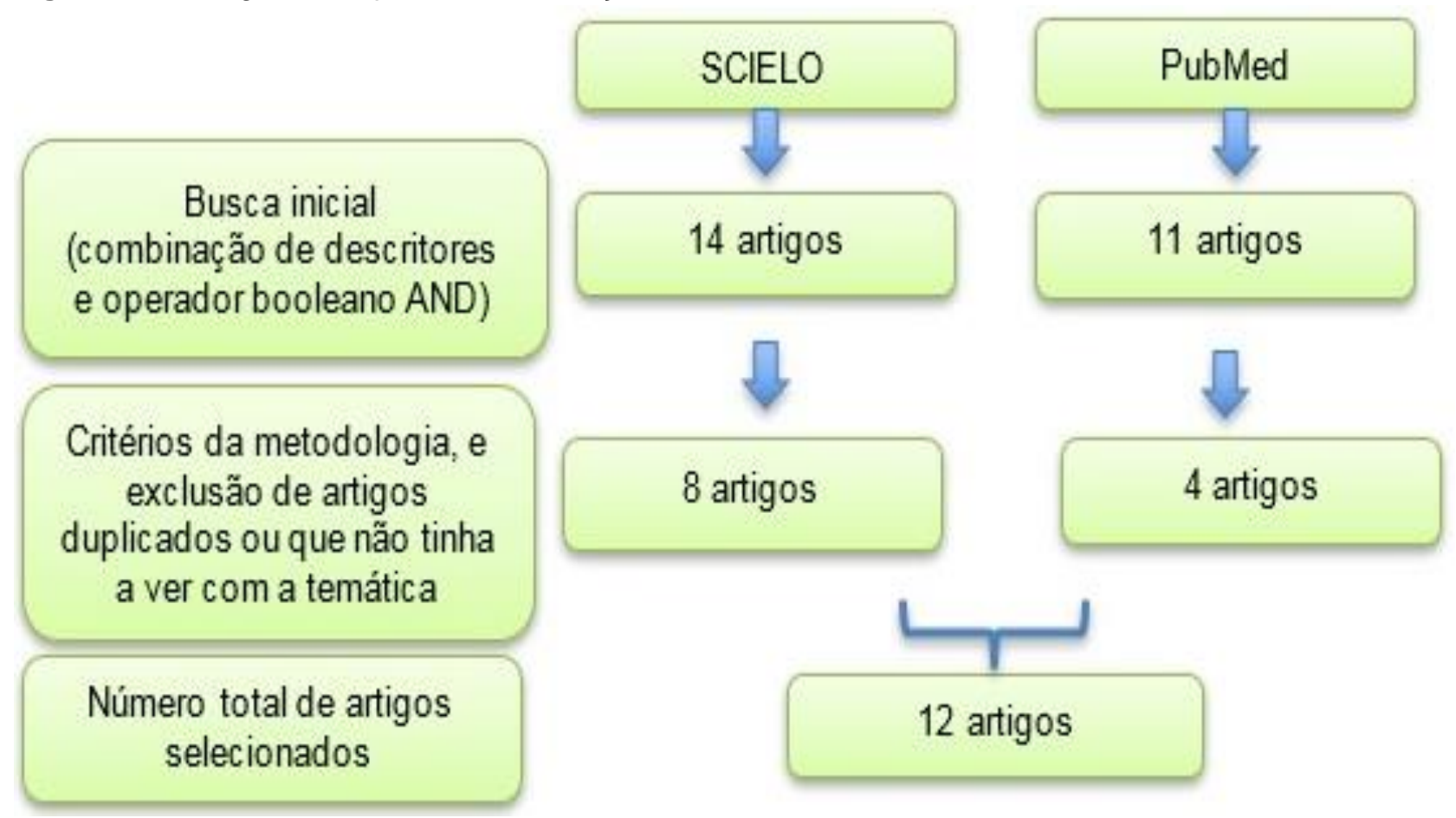

Fonte: Leite SCMC, et al., 2020.

Os artigos selecionados foram organizados em quadros sinópticos, com destaque das vertentes autor e ano de publicação, base de dados proveniente, objetivo e principais resultados. Os dados coletados foram comparados com outros autores, algumas leis e portarias, e divididos em categorias para melhor discussão da temática.

\section{RESULTADOS E DISCUSSÃO}

Foram selecionados 12 artigos provenientes das bases SCIELO e PubMed, em sua maioria os estudos enfatizaram a telemedicina e a relação médico-paciente, e também a influência da telemedicina na assistência, uma vez que em meio a pandemia de Covid-19 os canais e mídias de saúde são cada vez mais utilizados (Quadro 1). 
Quadro 1 - Caracterização dos artigos selecionados e seus principais resultados, $\mathrm{n}=12$.

\begin{tabular}{|c|c|c|c|}
\hline $\begin{array}{l}\text { Autores/Ano de } \\
\text { publicação }\end{array}$ & $\begin{array}{l}\text { Base de dados } \\
\text { proveniente }\end{array}$ & Objetivo & Principais resultados \\
\hline $\begin{array}{l}\text { LINS AF, et al., } \\
\qquad 2019\end{array}$ & SCIELO & $\begin{array}{l}\text { Discutir a viabilidade da telemedicina abordando } \\
\text { seus prós e contras. }\end{array}$ & $\begin{array}{l}\text { A telemedicina proporciona significativa economia de dinheiro, tempo } \\
\text { e distância, e igualdade de informações e assistência à saúde em } \\
\text { locais remotos. }\end{array}$ \\
\hline $\begin{array}{l}\text { MALDONADO } \\
\text { JMSV, et al., } 2016\end{array}$ & SCIELO & $\begin{array}{l}\text { Identificar as principais iniciativas já em curso de } \\
\text { desenvolvimento da telemedicina no Brasil. }\end{array}$ & $\begin{array}{l}\text { A telemedicina promove uma maior integração da relação médico- } \\
\text { paciente, superando a fragmentação ainda presente para a } \\
\text { efetivação do direito integral à saúde. }\end{array}$ \\
\hline $\begin{array}{l}\text { PINTO JA, et al., } \\
2012\end{array}$ & SCIELO & $\begin{array}{l}\text { Buscar o perfil da relação médico-paciente na } \\
\text { percepção do paciente. }\end{array}$ & $\begin{array}{l}\text { A relação médico-paciente descrita no estufo foi boa, pautada de } \\
\text { atributos como empatia, humildade, serenidade, respeito com as } \\
\text { diferenças. }\end{array}$ \\
\hline $\begin{array}{l}\text { BITTENCOURT } \\
\text { ALP, et al., } 2013\end{array}$ & SCIELO & $\begin{array}{l}\text { Compreender que fatores levaram os participantes a } \\
\text { identificarem coerção ao expressarem-se em } \\
\text { relação ao seu tratamento. }\end{array}$ & $\begin{array}{l}\text { O descontentamento com a falta de informação recebida do médico } \\
\text { levou os entrevistados ao sentimento de terem sido abandonados. }\end{array}$ \\
\hline $\begin{array}{l}\text { SOUZA YV, et al., } \\
\qquad 2020\end{array}$ & SCIELO & $\begin{array}{l}\text { Conhecer a percepção de } 200 \text { pacientes na cidade } \\
\text { de Aracaju/SE sobre sua relação com os médicos. }\end{array}$ & $\begin{array}{l}\text { Um ambiente favorável para a boa relação médico-paciente depende } \\
\text { não apenas da infraestrutura clínico-hospitalar, mas também da } \\
\text { habilidade comunicativa dos profissionais. }\end{array}$ \\
\hline $\begin{array}{l}\text { DIMER NA, et al., } \\
\qquad 2020\end{array}$ & SCIELO & $\begin{array}{l}\text { Analisar a influência da pandemia do COVID-19 e } \\
\text { implementação de telefonoaudiologia para pacientes } \\
\text { em domicílio. }\end{array}$ & $\begin{array}{l}\text { A telessaúde tem se mostrado um recurso eficiente para atendimento } \\
\text { de pacientes, possibilitando o atendimento remoto com a mesma } \\
\text { qualidade que o atendimento presencial. }\end{array}$ \\
\hline
\end{tabular}

REAS/EJCH | Vol.13(1) | e5694 | DOI: https://doi.org/10.25248/reas.e5694.2021 Página 4 de 9 
Revista Eletrônica Acervo Saúde / Electronic Journal Collection Health | ISSN 2178-2091

\begin{tabular}{|c|c|c|c|}
\hline $\begin{array}{l}\text { Autores/Ano de } \\
\text { publicação }\end{array}$ & $\begin{array}{l}\text { Base de dados } \\
\text { proveniente }\end{array}$ & Objetivo & Principais resultados \\
\hline $\begin{array}{l}\text { KNORST GRS, et } \\
\text { al., } 2019\end{array}$ & SCIELO & $\begin{array}{l}\text { Evidenciar possibilidades na relação do médico } \\
\text { com paciente expert. }\end{array}$ & $\begin{array}{l}\text { O empoderamento do paciente expert perante a autoridade do } \\
\text { médico ainda permanece questionável, sobretudo quando se trata } \\
\text { da redistribuição do poder que emana do saber. }\end{array}$ \\
\hline LUZ PL, 2019 & SCIELO & $\begin{array}{l}\text { Analisar a influência da telemedicina na relação } \\
\text { médico-paciente. }\end{array}$ & $\begin{array}{l}\text { A telemedicina se mostrou eficaz para o aprimoramento da relação } \\
\text { médico-paciente, uma vez que preserva o humanismo na medicina } \\
\text { e usa as novas tecnologias para aperfeiçoar o cuidado médico. }\end{array}$ \\
\hline $\begin{array}{l}\text { ROCHA BV, et al., } \\
\qquad 2011\end{array}$ & PubMed & Analisar a construção da relação médico-paciente. & $\begin{array}{c}\text { A relação médico-paciente é de difícil construção, é um processo } \\
\text { complexo que demanda esforço de ambas as partes e não se limita } \\
\text { às paredes dos hospitais e consultórios. }\end{array}$ \\
\hline $\begin{array}{l}\text { CAETANO R, et al., } \\
\qquad 2020\end{array}$ & PubMed & $\begin{array}{c}\text { Verificar os desafios e oportunidades para } \\
\text { telessaúde em tempos da pandemia pela COVID- } \\
19 .\end{array}$ & $\begin{array}{c}\text { A telessaúde oferece capacidades para o cuidado e tratamento } \\
\text { remotos, auxilia o monitoramento, detecção e prevenção dos } \\
\text { problemas de saúde. }\end{array}$ \\
\hline $\begin{array}{l}\text { SILVESTRE JCC, } \\
\quad \text { et al., } 2012\end{array}$ & PubMed & $\begin{array}{c}\text { Analisar o uso da internet pelos pacientes como } \\
\text { fonte de informação em saúde e a sua influência na } \\
\text { relação médico-paciente. }\end{array}$ & $\begin{array}{l}\text { O uso de informações da internet não prejudica a relação médico- } \\
\text { paciente. }\end{array}$ \\
\hline $\begin{array}{l}\text { GONÇALVES AA, } \\
\text { et al., } 2019\end{array}$ & PubMed & $\begin{array}{c}\text { Descrever a utilização da Telemedicina na } \\
\text { divulgação das informações para o atendimento e } \\
\text { tratamento de pacientes no Instituto Nacional do } \\
\text { Câncer. }\end{array}$ & $\begin{array}{c}\text { A telemedicina vem mudando a relação médico-paciente, e ambos } \\
\text { devem confiar no uso da telemedicina. A confiança é um requisito } \\
\text { importante para a adoção de inovação. }\end{array}$ \\
\hline
\end{tabular}

Fonte: Leite SCMC, et al., 2020.

REAS/EJCH | Vol.13(1) | e5694 | DOI: https://doi.org/10.25248/reas.e5694.2021 Página 5 de 9 
Com base nos achados, foram organizadas quatro categorias para melhor discussão da temática, sendo elas: Relação médico paciente na atualidade; Os benefícios e obstáculos em relação ao atendimento por meio da telemedicina; Difusão da telemedicina no Brasil; Relação da pandemia com a telemedicina atualmente.

\section{Relação médico paciente da atualidade}

De acordo com Souza YV, et al. (2020) a relação médico-paciente está cada vez mais em foco na sociedade atual, com a criação de leis sobre a humanização em saúde, e estudos sobre a mecanização do profissional de medicina, é inaceitável que o médico apenas olhe o paciente com um ser portador de uma doença, atualmente é preconizado que a saúde abranja aspectos físicos, sociais e emocionais, assim paciente é um ser complexo que exige complexidade no tratamento, que tenha uma assistência de qualidade e que seja tratado com dignidade.

Corroborando com a afirmação anterior, Silvestre JCC, et al. (2012), afirmaram ainda que a relação médico-paciente não pode ser vista apenas como uma situação imprevisível, os autores explanaram que vai muito além do que a maioria da população pensa, pois vai muito além de uma simples anamnese e de um exame físico, é uma relação que necessita da sensibilidade humana, da empatia pelo próximo dentro de todo o contexto em que o profissional e o paciente está inserido, não é apenas receitar medicamentos e prescrever condutas ela precisa aptidões técnicas e pessoais.

Para Caprara A e Rodrigues J (2004), o assunto referente à relação médicos e pacientes não é uma novidade para a profissão médica, pois sempre foi necessário que o paciente confiasse no medico para seguir o tratamento, além disso, os autores mostraram que desde o início do século 20 este conteúdo vem sendo apresentado, e o próprio contexto da medicina abrange esta questão como um sinal indicador dos processos de alterações a que se sujeita.

Segundo Rocha BV, et al. (2011), existem na atualidade dois tipos de relação médico-paciente, uma se refere ao atendimento nos hospitais e clínicas pautados na relação presencial, já a outra tem como ponto principal a longitudinalidade, uma relação continuada ofertada na atenção primaria à saúde. Portanto, para se ter uma relação prazerosa entre o médico e o paciente é imprescindível que o profissional se encaixe no lugar do paciente, e só assim será capaz de concernir toda a situação da pessoa que está se consultando e com isso, garantir uma medicina mais humanizada e utilizar as tecnologias para favorecer ainda mais esse cuidado médico com o paciente.

Ainda de acordo com Rocha BV, et al. (2011), a relação médico-paciente evoluiu das abordagens utilizadas pelos profissionais, devendo exprimir as características humanas subjetivas, de uma forma natural. Além disso, os autores destacam que essa relação é um aspecto chave para a melhoria da qualidade do serviço que tem como objetivo uma assistência mais humanizada no qual os pacientes têm o direito à informação, assim como o consentimento informado, podendo dar sua opinião sobre o tratamento.

Nessa perspectiva Bittencourt ALP, et al. (2013) reforçaram em seu estudo que com o passar dos anos a medicina sofreu muitas modificações, tais modificações puderam ser vistas através das melhorias das rotinas hospitalares e da evolução dos tratamentos, tais fatores culminaram diretamente na modificação da relação do médico com o paciente, pois esse passou a buscar por se próprio o conhecimento sobre a saúde, e questionar o profissional de medicina quando acha necessário.

Dessa forma, o paciente passou a ter uma atitude mais ativa em relação ao que diz respeito o tipo de tratamento e todas as informações que ele tem direito de ter acesso, mas esse fato não influenciou negativamente na relação médico-paciente, pelo contrário, fez com que os médicos se interessassem ainda mais em sanar as dúvidas de seus clientes.

Pinto JA, et al. (2012), expuseram que na atualidade a qualidade da relação médico-paciente é fundamental para uma melhor adesão e resultados mais eficazes dos tratamentos, pois influencia diretamente o equilíbrio do processo saúde-doença e a promoção de saúde, para tal é necessário que o profissional de medicina tenha empatia pela situação de saúde vivenciada pelo paciente, saiba escutar as queixas do paciente sem julgamento, e procure ser o mais simpático e respeitoso possível. 


\section{Benefícios e obstáculos do atendimento por meio da telemedicina}

A telemedicina é conceituada por Gonçalves AA, et al. (2019), como as atividades à distância realizada pelos profissionais de saúde por meio das tecnologias da informação e telecomunicações (TICs) que proporciona um melhor acesso aos pacientes em casa ou em organizações de cuidados primários, aumentando o acesso ao cuidado especializado, reduzindo os problemas devido à dispersão geográfica $\mathrm{e}$ melhorando a qualidade de vida dos cidadãos.

Os autores afirmaram ainda que o conceito de telemedicina pode variar devido às funções, envolvimentos institucionais e profissionais, o contexto e os objetivos que são preconizados. Segundo Barbosa PHFA, et al. (2019), telemedicina ao ser utilizada precisa ter uma preparação e estruturação adequada para se obter êxito, pois é importante que a telemedicina seja planejada para evitar imprevistos ou falhas técnicas ao ser utilizada na companhia do paciente.Além disso, uma relação harmoniosa entre os atores que fazem uso das tecnologias, tais como os profissionais da saúde e os pacientes, se tornam imprescindíveis para o sucesso desse serviço, e não se pode deixar de considerar as questões individuais de cada local para atingir o resultado esperado para aquela sociedade.

Contudo, Knorst GRS, et al. (2019), explicaram que com a ampliação da telemedicina se tornaram possíveis atualmente a realização de teleconsultorias, teleconsultas, telediagnósticos, telecirurgias e telemonitoramento, garantindo ao paciente um acesso a saúde de maneira prática e acessível, visto que algumas regiões no Brasil sofrem com escassez de profissionais de medicina. Frente a isso, Gonçalves AA, et al. (2019), explanaram que a implementação da telemedicina traz alguns pontos positivos tanto para os profissionais como para os pacientes usuários do serviço, pois reduz os custos na medicina e junto à isso, aumenta a acessibilidade ao sistema de saúde, principalmente nas áreas mais remotas e carentes.

Conforme o raciocínio reflexivo de Maldonado JMSV, et al. (2016), é notório que com o advento da globalização, a comunicação de toda humanidade avançou de forma bastante satisfatória, pois a facilidade de se comunicar com o auxílio de aparelhos tecnológicos ampliaram o acesso a bens e serviços, e a partir disso houve também uma facilitação dos serviços na área da saúde, especialmente para os profissionais que precisavam se relacionar com pessoas diariamente, como é o caso da medicina, e que em algumas situações não tinham como entrar em contato com o paciente. Assim o uso de mídias quebrou barreiras e aproximou o paciente e os profissionais de saúde, quebrando as barreiras do consultório médico, para que a saúde possa ser acessada sempre que necessário.

Tangente a isso, com o acesso remoto o princípio da igualdade material, basilar na Constituição Federal de 1988, é alcançado através desse sistema de atendimento, com isso Lins AF, et al. (2019) afirmaram que as pessoas que vivem em locais de difícil acesso em vários lugares do país tem a possibilidade de acesso a consultas sem o deslocamento físico, onde traz maior eficiência ao serviço de saúde. Todavia, os autores explanaram ainda que apesar da facilidade e agilidade de atendimento médico, surge um problema ao se utilizar a telemedicina como meio de serviço, pois a qualidade do atendimento é um fator a ser questionado já que o contato presencial é necessário para a prescrição de medicamentos adequados, pois o profissional atuante não tem o acesso ao prontuário do paciente.

Portanto Lins AF, et al. (2019), e Maldonato JMSV, et al. (2016) mostraram como existem vários pontos positivos e negativos a serem discutidos e analisados de forma bastante crítica, pois ao passo que com o auxílio da telemedicina o atendimento traz uma maior economia de tempo e dinheiro para ambos os lados, médico e paciente, existe muitas vezes uma diminuição na qualidade do serviço na qual a visão conservadora tem dificuldade de transição do atendimento presencial para o remoto.

\section{Difusão da telemedicina no Brasil}

Maldonato JMSV, et al. (2016), afirmaram que no Brasil a difusão da telemedicina ocorreu devido diversas iniciativas políticas governamentais, tais como o Programa Telessaúde Brasil que foi instituído pelo Ministério da Saúde em 2007, e ampliado em 2011 para o Programa Nacional Telessaúde Brasil Redes com foco na atenção primária presente em 23 estados brasileiros, funcionando e evoluindo de acordo com a necessidade de cada estado. 
Além disso, foram expostas outras iniciativas governamentais como a elaboração da Rede Nacional de Ensino e Pesquisas (RNP) que criou o projeto Rede Universitária de Telemedicina (RUTE) que tinha como objetivo principal à construção de uma infraestrutura de rede de Internet nacional no âmbito acadêmico como a interconexão nos hospitais universitários e as instituições de ensino de saúde do Brasil.

O Ministério da Saúde em 29 de dezembro de 2014 publicou a portaria ํำ 2.859, onde estabeleceu o incentivo financeiro a implantação da Telessaúde estaduais e intermunicipais. Portanto, esse incentivo teve como finalidade melhorar a qualidade dos serviços da atenção básica, principalmente as Equipes de Saúde da Família, e oferecer meios de igualdade na distribuição dos serviços de saúde, especialmente as regiões mais necessitadas, onde o número de profissionais de saúde não é eficaz para atender a demanda populacional (MINISTÉRIO DA SAÚDE; 2014).

Além disso, Maldonato JMSV, et al. (2016) explanaram que se deve levar em consideração o fato de que existe uma interdependência entre o potencial da telemedicina e a organização dos serviços de saúde. Assim as mudanças variam de acordo com a disposição e locais em que são oferecidos esses serviços, além de fatores como a agilidade de atendimento, a redução dos custos, e a facilidade de atendimento devido às dificuldades físicas de acesso por parte da população.

Contudo, o Ministério da Saúde tentou reduzir ao máximo a utilização da telemedicina por concluir que tal atividade configurava descumprimento ético uma vez o profissional de medicina não poderia prescrever tratamento ou outros tipos de procedimentos sem exame físico direto do paciente. Entretanto, com a pandemia da COVID-19, o contexto da telemedicina configurou mudanças com o Projeto de Lei ํo 696/2020, que permitiu o uso da telemedicina em todo ambiente da área da saúde no Brasil, e a Lei no 13.989/2020, que fala da regulamentação da telemedicina depois do término da pandemia e também passou a validar receitas digitais que possuem certificação digital (BRASIL, 2020).

\section{Relação da pandemia da covid-19 com a telemedicina}

Segundo Dimer NA, et al. (2020), a pandemia de COVID-19 acarretou diversos desafios, que antes eram imprevisíveis para o século $\mathrm{XXI}$, especialmente em relação à reorganização dos indivíduos a sua rotina familiar, lazer e consumo tanto de bens quanto de serviço, tais como serviços de saúde. Assim as instituições de saúde passaram a ser evitadas pelas pessoas, especialmente aquelas que se encaixam como grupo de risco para a COVID-19.

De acordo com Caetano $\mathrm{R}$, et al. (2020) a telessaúde é um meio indispensável, pois está habilitada à amenizar a circulação de indivíduos nas instituições, diminuir a propagação e a exposição à infecção da COVID-19, permite uma maior acessibilidade no contexto geográfico e ajuda na disseminação do serviço de saúde para a sociedade que está impossibilitada de comparecer nessas instituições. Além disso, os autores expuseram que no contexto social, a telemedicina propicia uma resolução inovadora na prestação de serviços de saúde devido à sua versatilidade e intuito com as tecnologias digitais.

Dimer NA, et al. (2020) afirmaram que o objetivo primordial e necessário do uso da tecnologia nos processos de disseminação de saúde é de ampliar o acesso aos cuidados em saúde. Além disso, os autores expuseram, que com a utilização da telemedicina ocorreu um aumento considerável no número de consultas em comparação com o atendimento presencial, isso se deve ao diversos fatores de impedimento como a falta de transportes, a indisponibilidade de horários, e o incomodo de se deslocar a um local.

Segundo Luz PL. (2019) a quarentena associada ao medo populacional em sair de casa e se expor a locais com maior risco de infecção como, por exemplo, os estabelecimentos de saúde, fez com que a telemedicina se sobressaísse e fosse uma modalidade bastante procurada atualmente pela população, sendo mais visada até do que os cuidados home care, pois a telemedicina permite o distanciamento físico entre o paciente e o médico, porém culminou para a aproximação da relação médico-paciente, pois forneceu meios para conversa e escuta qualificada, longe do estresse de filas que comumente são vistas nos estabelecimentos de saúde, e que fazem com que os médicos façam uma consulta rápida e superficial na maioria dos casos. 
Contudo, Caetano R, et al. (2020), apresentaram em seu estudo que o uso da telessaúde oferece capacidades para impugnar a COVID-19, e torna o atendimento mais seguro tanto para o profissional de saúde como para o paciente. Além disso, expuseram a importância de manter todos os direitos do paciente de ter o tratamento mais adequado as suas necessidades, visando ofertar um atendimento justo e competente, assim como na rotina de atendimento nos estabelecimentos de saúde, optando em realizar os atendimentos com técnicas respaldadas por evidência científica.

\section{CONSIDERAÇÕES FINAIS}

A telemedicina possui influência positiva perante a relação médico-paciente, uma vez que através da comunicação eficaz e da escuta qualificada o paciente se sente mais confiante para seguir as recomendações médicas, tendo ainda benefícios como a redução dos custos hospitalares e aumento da acessibilidade ao sistema de saúde. Conclui-se que a telemedicina é uma ferramenta que contribui para a aproximação do médico com o paciente, especialmente em tempos de pandemia, como a da COVID-19, em que o contato físico social teve que ser restringido.

\section{REFERÊNCIAS}

1. ALMEIDA JP, et al. Telemedicina e bioética: o futuro é agora. Revista bioética CREMEGO, 2019; 1(1): 41-45.

2. ARANTES ACC, et al. Usabilidade da telemedicina como uma plataforma de ensino Colaborativo para estudantes de medicina. Jornal Brasileiro de TeleSaúde, 2013; 2(4): 79-85.

3. BARBOSA PHFA, et al. Telemedicina. Novas tecnologias aplicadas à saúde: desenvolvimento de sistemas dinâmicos: conceitos, aplicações e utilização de técnicas inteligentes e regulação. EDUERN, 2019; 15(6).

4. BATISTA NA, LESSA SS. Aprendizagem da Empatia na Relação Médico-Paciente: um Olhar Qualitativo entre Estudantes do Internato de Escolas Médicas do Nordeste do Brasil. Revista Brasileira de Educação Médica, 2019; 43(1): 349-356.

5. BRASIL. Lei no 13.979, de 6 de fevereiro de 2020. Dispõe sobre as medidas para enfrentamento da emergência de saúde pública de importância internacional decorrente do coronavírus responsável pelo surto de 2019. Diário Oficial da União, 2020.

6. BITTENCOURT ALP, et al. A voz do paciente: por que ele se sente coagido? Psicologia em Estudo, 2013; 18(1): 93101.

7. CAETANO R, et al. Desafios e oportunidades para telessaúde em tempos da pandemia pela COVID-19: uma reflexão sobre os espaços e iniciativas no contexto brasileiro. Cadernos de Saúde Pública, 2020; 36(5).

8. CAPRARA A, RODRIGUES J. A relação assimétrica médico-paciente: repensando o vínculo terapêutico. Ciência \& saúde coletiva, 2004, 9(1): 139-146.

9. DIMER NA, et al. Pandemia do COVID-19 e implementação de telefonoaudiologia para pacientes em domicílio: relato de experiência. CoDAS, 2020; 32(3).

10. GONÇALVES AA, et al. Impactos da implantação da Telemedicina no Tratamento e Prevenção do Câncer. Revista Ibérica de Sistemas e Tecnologias de Informação, 2019; 17(3): 222-230.

11. KOGA RCR, et al. Telemedicina e sua relação com comunicação, tecnologia e convergência. Revista Arquivos Científicos (IMMES), 2010; 3(1): 111-116.

12. KNORST GRS, et al. A relação com o médico na era do paciente expert: uma análise epistemológica. Interface, 2019; 23(5): 180-193.

13. LINS AF, et al. O uso da telemedicina como ferramenta para aprimorar os serviços de saúde: viabilidade e desafios. Revista Educação em Saúde, 2019; 7(12).

14. LUZ PL. Telemedicina e a Relação Médico-Paciente. Arquivos Brasileiros de Cardiologia, v. 113, n. 1, p. 100-102, 2019. Telemedicina e a Relação Médico-Paciente. Arquivos Brasileiros de Cardiologia, 2019; 113(1): 100-102.

15. MALDONADO JMSV, et al. Telemedicina: desafios à sua difusão no Brasil. Cadernos de Saúde Pública, 2016; 32(2):155-161.

16. MINISTÉRIO DA SAÚDE. Portaria no 2.859, de 29 de dezembro de 2014. Institui o incentivo financeiro de custeio mensal destinado aos Núcleos Intermunicipais e Estaduais de Telessaúde do Programa Nacional de Telessaúde Brasil Redes na Atenção Básica, e dá outras providências. Diário Oficial da União 2014.

17. PINTO JA, et al. A Relação Médico-Paciente Segundo a Perspectiva do Paciente. Revista Ciências em Saúde, 2012; 2(3): 17-27.

18. ROCHA BV, et al. Relação médico-paciente. Revista do médico residente, $2011 ; 13(2)$.

19. SANTANA S, PEREIRA AS. Da utilização da internet para questões de saúde e doença em Portugal: possíveis repercussões na relação médico-doente. Acta Med Port, 2007; 20(1): 47-57.

20. SILVESTRE JCC, et al. Uso da internet pelos pacientes como fonte de informação em saúde e a sua influência na relação médico-paciente. Revista AMRIGS, 2012; 23(4): 149-155.

21. SOUZA YV, et al. Percepção de pacientes sobre sua relação com médicos. Revista de Bioética de Brasília, 2020; 28(2): 332-343. 